\title{
12. THE NUCLEI OF PLANETARY NEBULAE AS PROGENITORS OF WHITE DWARFS
}

\author{
C. R. O'DELL \\ Yerkes Observatory, Williams Bay, Wis., U.S.A.
}

Stellar evolution is characterized by fast and slow phases. Usually the periods of rapid change are difficult to follow observationally; but, this does not seem to be the case when passing through the planetary nebula stage. Because of their high intrinsic luminosities and easy identification, it is possible to identify and study these objects and their central stars rather completely. It is quite relevant to discuss these objects at a symposium on white dwarfs since the central stars may be in the immediate progenitor stage before white dwarfs. The actual picture of the evolution of the nuclei has changed rather little in the past few years and is the subject of an earlier review article (O'Dell, 1968) to which the reader is referred.

In capsule form, the evolutionary picture is as follows, the systems are discovered soon after ejection of a shell of about 0.3 solar masses and the stars have luminosities about $10^{4}$ solar luminosities. The stars seem to increase in temperture to about $10^{5} \mathrm{~K}$ at constant luminosity, then to decrease in luminosity very quickly, falling to ten solar luminosities in a total time of about $30000 \mathrm{yr}$, placing them at the threshold of the hot extension of the white dwarf cooling tracks. It is a suggestive feature of this picture that the luminosities peak close to the radiation pressure boundary for stars of about one solar mass, thus providing an efficient mechanism for mass loss.

There are complications to the above picture that may actually provide fundamental clues to the exact mechanisms occurring. Perhaps most puzzling is the question of multiple ejections of material. This may be evidenced by the double nebula structure of NGC 6543 and NGC 6826. They give every appearance of shell formation at epochs about $30000 \mathrm{yr}$ apart. A photographic search of the brightest dozen other nebulae adds no others to this list. Another such object may be FG Sagittae (Herbig and Boyarchuk, 1968), which is a nebula with a central star of quite cool spectral type. The system is extremely interesting, because the central star is cooling and increasing in luminosity. Herbig and Boyarchuk believe that the absorption spectrum is actually formed in an expanding atmosphere which will become a second shell. It is not at all clear whether the small fraction of double shells should be interpreted as being due to long intervals between ejection or that only a small fraction of the stars create double shells.

Perhaps one of the best methods of testing the model and the several theories would be through the determination of the gravitational redshifts, which yield values of Mass/Radius. Such a program is reasonable because the radial velocity can be obtained from the optically thin nebular shell. The problems due to faintness and weak, broad absorption lines are serious, but not intractable. 
The vital statistics for these objects are given in the table below, where the white dwarf data for the rate of formation $(x)$ are due to Weideman (1968). From these numbers, we see that we can account for about $20 \%$ of all white dwarfs. The errors in $\boldsymbol{x}$ could allow this fraction to approach $80 \%$. The safe conclusion is that one can account for a substantial fraction of all white dwarfs by stars manifesting the planetary nebula phenomenon.

Density near sun

Formation Rate $(\boldsymbol{x})$

\section{Planetaries}

$1.4 \times 10^{-8} \mathrm{pc}^{-3}$

$0.4 \times 10^{-12} \mathrm{pc}^{-3} \mathrm{yr}^{-1}$
White dwarfs

$$
10^{-2} \mathrm{pc}^{-3}
$$$$
2 \times 10^{-12} \mathrm{pc}^{-3} \mathrm{yr}^{-1}
$$

\section{References}

Herbig, G. H. and Boyarchuk, A. A.: 1968, IAU Symp. 34, p. 383, in D. E. Osterbrock and C. R. O'Dell (eds), Planetary Nebulae, Reidel, Dordrecht.

O'Dell, C. R.: 1968, IAU Symp. op. cit., p. 361.

Weideman, V.: 1968, IAU Symp. op. cit., p. 423. 